Vietnam Journal of Mechanics, VAST, Vol. 27, No. 3 (2005), pp. 158-170

\title{
INSTABILITY BEHAVIOR OF ARCHES UNDER QUASI-STATIC CONCENTRATED LOAD
}

\author{
NGUYEN Dinh KIEN \\ Institute of Mechanics
}

\begin{abstract}
The present paper investigates the instability characteristics of arch structures under quasi-static concentrated load. The arches are idealized as equivalent frameworks and analyzed by the finite element method using the nonlinear co-rotational beam elements. The numerical algorithms for computing the limit load and tracing the equilibrium path are presented. In particular, the automatic cutting and automatic increment techniques needed for the incremental/iterative procedure are described in detail. The effects of geometry, boundary condition, shear deformation, loading condition as well as the material characteristics on the instability behavior of both the elastic and elasto-plastic arches are numerically investigated.
\end{abstract}

\section{INTRODUCTION}

Arch structures play an important role in civil engineering, and basic arch constructions have been known and used successfully for centuries. The analysis and design of arches based on the concepts of linearities have been described in some textbooks $[1,2]$. However, with the invention of new material the arches can be designed more slender and withstanding more loads. Consequently, the instability problem of arches has been received much attention in the field of structural mechanics.

From practical point of view, most instability phenomena happen under quasi-static loads, in which a considerable change in the structural geometry is occurred with a steady increase in the external load. Furthermore, nearly all the steel arches that have been built lie in vertical plane [2]. Accordingly, a 2D analysis can be employed when the external loads also act in the vertical plane. The present work assumes the arch is loaded by quasistatic concentrated loads in its plane, so that the instability analysis in the vertical plane can be adopted.

In the context of the finite element method, a nonlinear arch element (also known as curved beam element in the literature) is necessary to formulate for the instability analysis of arches, and the work in this line has been carried out by some authors such as Wood and Zienkiewicz [3], Xu and Mirmiran [4]. However, in the mesh refinement, a arch element goes back to a straight arch element which is identical to a beam element [5]. In the above regard, the nonlinear beam elements such as the co-rotational elements, previously formulated by the author for large deflection analysis of frames, can be employed to analyze the instability phenomena of arches by idealizing the arches as equivalent frameworks. This method has been applied by Hsiao and Huo in nonlinear analysis of arches [6], and will be again adopted in this paper.

Adopting the co-rotational elements, the main focus of the paper is developing the suitable numerical algorithms needed for computing the equilibrium path and limit point of the arches. It is necessary to mention that the numerical algorithms previously used by the author in the large deflection analysis of frames are not sufficient, and sometimes not suitable for analyzing arch structures, so that the new techniques are required. 

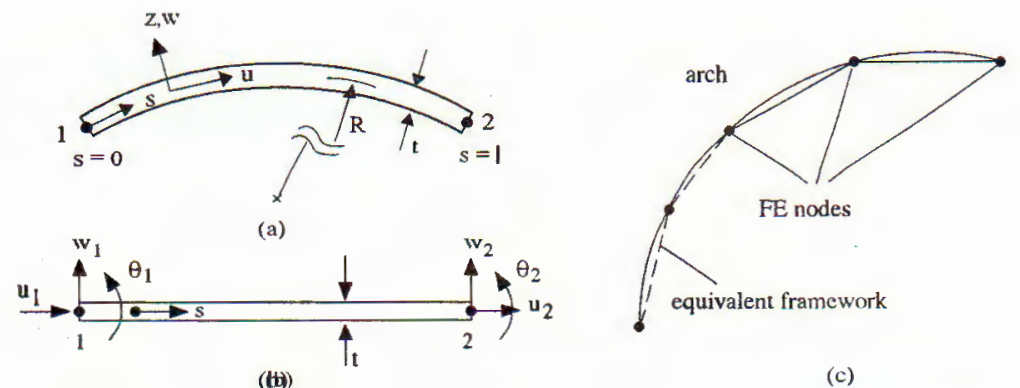

Fig. 1. Equivalent framework arch: a curved arch element (a), and its straight counterpart (b); arch discretzed by straight arch elements (c).

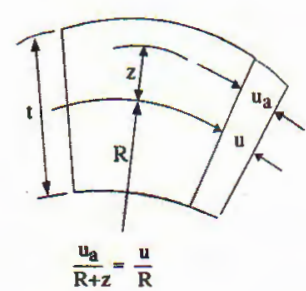

(a)

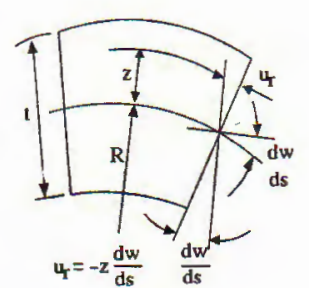

(b)

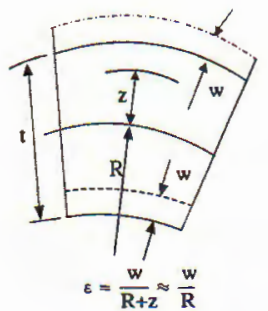

(C)

Fig. 2. Kinematics of arch element: axial and rotational displacements $(a, b)$; radial strain (c).

Following the above introduction, the remainder of this paper is organized as follows: Section 2 gives the basic equations and kinematics for an arch element, so that we could understand how an arch element becomes a beam element in the mesh refinement. Section 3 summarizes the co-rotational beam elements, previously formulated by the author and will be employed in this paper for the analysis. Section 4 discusses the numerical algorithms. The numerical investigations are presented in Section 5. Finally, the main conclusions of the paper are given in Section 6 .

\section{ARCH AS EQUIVALENT FRAMEWORK}

The concept of equivalent framework arch mentioned in the previous Section is depicted in Fig. 1, where an arch is modelled by straight arch elements with 6 degrees of freedom. Below we show the similarity between an arch element and a beam element, and in the mesh refinement the arch element goes back to the beam element.

Consider an arch element with constant mean radius $R$ and arc length of $l$ as depicted in Fig. 1a. A point on the midline has $s$-direction (tangential) displacement $u$ and $z$ direction (radial) displacement $w$. Denoting $\epsilon_{s}$ the tangential strain at a point distanced $z$ from the midline. Using the kinematics shown in Fig. 2, we can write

$$
\epsilon_{s}=\frac{d\left(u_{a}+u_{r}\right)}{d s}+\frac{w}{R}=\frac{d u}{d s}+\frac{w}{R}+z\left(\frac{1}{R} \frac{d u}{d s}-\frac{d^{2} w}{d s^{2}}\right),
$$

where $u_{a}$ and $u_{r}$ are the axial and rotational displacements of the point, respectively. equation (2.1) can be rewritten in a familiar form as the axial strain of a beam element as

with

$$
\epsilon_{s}=\epsilon_{m}+z \chi
$$

$$
\epsilon_{m}=\frac{d u}{d s}+\frac{w}{R} \text { and } \chi=\frac{1}{R} \frac{d u}{d s}-\frac{d^{2} w}{d s^{2}}
$$


are the membrane strain and curvature of the element, respectively. In a similar way as the beam element, a higher order term can be added to equation (2.1) to improve the accuracy as

$$
\epsilon_{s}=\frac{d u}{d s}+\frac{w}{R}+\frac{1}{2}\left(\frac{d w}{d s}\right)^{2}+z\left(\frac{1}{R} \frac{d u}{d s}-\frac{d^{2} w}{d s^{2}}\right) .
$$

The above formulations have been derived by ignoring the effect of shear deformation which might be valid for thin arches. When the shear deformation is taken into account, in addition to the tangential and normal displacements of the midline, the arch element is described by the transverse shear rotation $\gamma(s)$, which related to the rotation of a normal to the midline by

$$
\gamma(s)=\frac{d w}{d s}-\theta(s)
$$

where $\theta(s)$ is the rotation of normal to the arch midline at the point having tangential coordinate $s$.

As seen from equation (2.4), when $R \longrightarrow \infty$, the tangential strain of the arch element is identical to the axial strain of a beam element, which previously employed by the author in formulating geometrically and materially nonlinear beam elements $[7,8]$. Thus, using the co-rotational approach, the nonlinear elements in $[7,8]$ can be employed to analyze the instability of arch structures by idealizing the arch as equivalent framework. When the shear deformation is taken into account, the element with shear deformation formulated in [9] should be employed.

\section{SUMMARY OF CO-ROTATIONAL BEAM ELEMENTS}

For the convenience of the reader, this Section summarizes some co-rotational nonlinear beam elements previously formulated by the author in $[7,8,9]$, and will be employed for the analysis below. The corotational approach is illustrated in Fig. 3, in which the deformation of the element is firstly captured in the local coordinates $\left(x_{l}, z_{l}\right)$, and then transferred to the global one $(x, z)$. Using the notations in Fig. 3 , the local and global vectors of nodal displacements are respectively given by

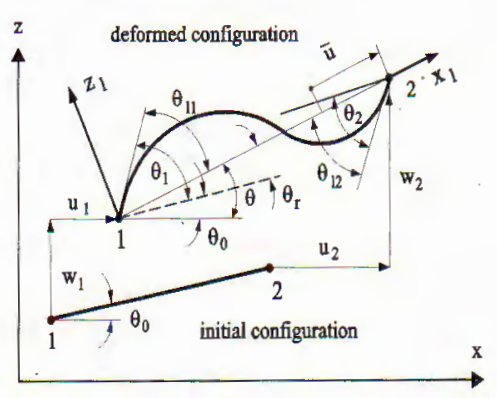

Fig. 3. Co-rotational beam element: global and local nodal displacements

$$
\begin{aligned}
\mathbf{d}_{l} & =\left\{\begin{array}{llll}
\bar{u} & \theta_{l 1} & \theta_{l 2}
\end{array}\right\}^{T}, \\
\mathbf{d} & =\left\{\begin{array}{llllll}
u_{1} & w_{1} & \theta_{1} & u_{2} & w_{2} & \theta_{2}
\end{array}\right\}^{T},
\end{aligned}
$$

where the subscript ' $l$ ' stands for the 'local'. Accordingly, the size of the local tangent stiffness matrix and internal force vector reduces to $(3 \times 3)$ and $(3 \times 1)$, respectively. In consequence, the element formulations are more simple, and the computations are markedly reduced. The local formulations are then transferred to the global system with the aid of the so-called transferred vectors or matrices describing the relationship between 
local and global degrees of freedom. Different types of element can be formulated based on the definition of the local axial strain.

\subsection{Elastic bernoulli beam element}

The elastic Bernoulli beam element based on the shallow arch theory formulated by the author in [7] shows many advantages in the accuracy and modelling ability. The local axial strain employed to develop the element has the form

$$
\epsilon_{x l}=\frac{\partial u_{l}}{\partial x_{l}}+\frac{1}{2}\left(\frac{\partial w_{l}}{\partial x_{l}}\right)^{2}-z_{l} \frac{\partial^{2} w_{l}}{\partial x_{l}^{2}}
$$

where $u_{l}$ and $w_{l}$ are the axial and transversal displacements measured in the local system, respectively. From equation (3.2), and with the elastic assumption, we can easily derive the strain energy $U$ for the element. Adopting linear interpolation function for the axial displacement and Hermite polynomiais for the local deflection, one can easily express $U$ in terms of the local nodal displacement $\bar{u}, \theta_{l 1}, \theta_{l 2}$. The element formulation can explicitly be obtained with the aid of symbolic software Maple [10] as

$$
\mathbf{f}_{l}=\operatorname{grad}\left(U,\left[\bar{u}, \theta_{l 1}, \theta_{l 2}\right]\right) \quad \text { and } \quad \mathbf{k}_{t l}=\operatorname{hessian}\left(U,\left[\bar{u}, \theta_{l 1}, \theta_{l 2}\right]\right) \text {. }
$$

For the convenience of latter discussion, the elastic beam element based on the local axial strain given by equation (3.2) is denoted B2ES.

\subsection{Elasto-plastic Bernoulli beam element}

The elasto-plastic analysis is required when the stress in the arch exceeds the yielding stress, which may be happened for the arch having low slenderness or for the arch constructed from the metal with low yielding stress. Using the concept of equivalent framework, this kind of arch can be analyzed by the elasto-plastic beam element presented in [8], in which a bilinear isotropic hardening material is adopted. The element is also formulated based on the local strain (3.2), but due to of plastic involvement, the Gauss quadrature is required to compute the tangent stiffness matrix and internal force vector, which are not able to derive explicitly. This elasto-plastic beam element is denoted B2PS.

\subsection{Beam element with shear deformation}

The beam element with shear deformation described in [9] is formulated by taking the effect of shear deformation into account. The advantage of this element lies on its ability in modelling the shear deformation effect. The shear deformation defined in (2.5) can be rewritten in the local coordinates as

$$
\gamma_{l}=\frac{\partial w_{l}}{\partial x_{l}}-\theta_{l}
$$

With the elastic assumption, the finite element formulations of this element can be derived explicitly following the same approach as B2ES element. The beam element including the shear strain (3.4) is denoted B2ET. 


\section{NUMERICAL ALGORITHMS}

As mentioned in Section 1, due to the particular geometry, the numerical procedures previously employed by the author for large deflection analysis of frames are not sufficient, and sometimes not suitable for the arch structures. This Section discusses the algorithms needed in computing the equilibrium path and the limit load of arches.

\subsection{Equilibrium and constraint equations}

The equilibrium equations describe the equilibrium between the nodal internal force vector and external force one, and have the form $[11,12]$

$$
\mathbf{R}(\mathbf{D}, \lambda)=\mathbf{F}_{\text {in }}-\mathbf{F}_{\text {ex }}=\mathbf{F}_{\text {in }}-\lambda \mathbf{f}_{\text {ex }}=\mathbf{0},
$$

where $\mathbf{R}(\mathbf{D}, \lambda)$ denotes the out of balance force vector, represented the difference between the internal force $\mathbf{F}_{\text {in }}$ and the external force $\mathbf{F}_{\text {ex }} ; \mathbf{D}$ is the vector of nodal displacements; $\lambda$ is the loading parameter; $\mathbf{f}_{\mathrm{ex}}$ is the fixed normalized external load vector. The system of nonlinear equations (4.1) can be solved by predictor-corrector procedure such as the Newton-Raphson method in which the norm of residual force vector is guided to zero.

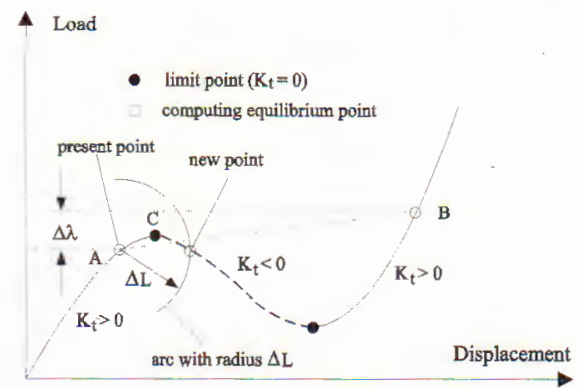

Fig. 4. The limit points and snap-through phenomenon in instability behavior

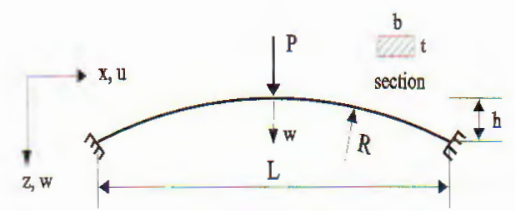

Fig. 5. Shallow arch under concentrated load at the crown, according to Ref. [19].

Previous work has shown that in the loading process the arch proceeds to a limit point, and then undergoes the snap-through phenomenon [3, 13], or even in complex looping behavior [4]. At a limit point, the structural tangent stiffness ceases to be positive definite, and then becomes negative definite beyond the limit point until a new limit point is reached. Such kind of behavior is depicted Fig. 4. Using the conventional iterative procedure under loading control leads to a dynamic jumping, in which the present equilibrium point $A$ (see Fig. 4) will follow the dot line to point $B$ after a load increment $\Delta \lambda$ [14]. For the looping and snap-back behavior, the similar problem is arisen if the traditional solution method is adopted, and new numerical algorithms which enable to trace the whole quasistatic equilibrium path are required. A procedure called 'spherical arc-length method' is adopted in this paper, in which the system of equilibrium equations (4.1) is supplemented by following constraint equation

$$
\dot{a}=\Delta \mathbf{D}^{T} \Delta \mathbf{D}+\Delta \lambda \psi^{2} \mathbf{f}_{\mathrm{ex}}^{T} \mathbf{f}_{\mathrm{ex}}=\Delta L^{2},
$$

where $\Delta L$ is the chosen scalar. For $\psi \neq 1$, equation (4.2) is the original constrain equation for the standard arc-length control method $[12,15]$. Choosing $\psi=1$, equation (4.2) describes a sphere in load-displacement space, which is adopted in the present work. 
Thus, instead of solving the system of equations (4.1), we are now looking for intersections of (4.1) with (4.2). It should be emphasized that in the present method the loading parameter $\lambda$ becomes a variable, hence together with $n$ displacement variables, we have a total of $(n+1)$ variables. An iterative procedure can be obtained by expanding equations (4.1) and (4.2) into Taylor series around the current equilibrium point, and having the form

$$
\left\{\begin{array}{c}
\delta \mathbf{D} \\
\delta \lambda
\end{array}\right\}=-\left[\begin{array}{cc}
\left.\mathbf{K}_{t}\right|_{0} & -\mathbf{f}_{e x} \\
2 \Delta \mathbf{D}^{T} & 2 \Delta \lambda \mathbf{f}_{e x}^{T} \mathbf{f}_{e x}
\end{array}\right]^{-1}\left\{\begin{array}{c}
\left.\mathbf{R}\right|_{0} \\
\left.a\right|_{0}
\end{array}\right\}=-\mathbf{J}^{-1}\left\{\begin{array}{c}
\left.\mathbf{R}\right|_{0} \\
\left.a\right|_{0}
\end{array}\right\},
$$

where $\left.(. .)\right|_{0$.$} denote the quantity (...) evaluated at 'old' equilibrium point. As seen from$ equation (4.3) the augmented stiffness matrix, or the Jacobian $\mathbf{J}$ in the square bracket remains non-singular even at the limit points where the tangent stiffness matrix $\mathbf{K}_{t}$ is singular, and the singularity of $\mathbf{J}$ at the limit point is avoided.

\subsection{Computing critical load}

The value of load at the limit point such as point $C$ in Fig. 4 is important from the design point of view, and we need to determine it. The bracketing procedure, previously discussed by the author in [16] for beams is adopted herewith, but with following caution.

A beam under axial force proceeds to a bifurcation point when the force approaches the Euler load. As a result, in loading process, the lowest eigenvalue denoted $\tau$ of the tangent stiffness $\mathbf{K}_{t}$ changes its sign when passing the bifurcation point, and we can use this characteristics as the indicator for the bracketing procedure (confirm [16]). The situation is different for arch, in which the structure comes a limit point, then follows the snap-through phenomenon such as in Fig. 4. If we increase the load, and monitor the lowest eigenvalue of $\mathbf{K}_{t}$, as in the case of beam, the path will trace the solid line until point $A$, and then follow the dot line to point $B$. As a result, the tangent stiffness matrix is always positive definite, and its lowest eigenvalue $\tau$ is always positive, and we fail to predict the limit point.

The situation can be overcome by monitoring the lowest eigenvalue of the tangent stiffness matrix in the arc-length procedure, not in the loading process. By doing this, we force the equilibrium path to follow the dash line (Fig.4), and $\mathbf{K}_{t}$ ceases to be positive definite when passing the limit point. As consequence, $\tau$ changes its sign from positive to negative. The bracketing procedure then being active to compute an immediate point using an interpolation scheme as [16]

$$
\lambda_{i}=\lambda_{l}-\frac{\lambda_{r}-\lambda_{l}}{\tau_{r}-\tau_{l}} \tau_{l}
$$

where $\lambda_{l}$ and $\lambda_{r}$ are the values of the loading parameter on the left and right sides of the limit point (before and after passing the limit point); $\tau_{l}$ and $\tau_{r}$ are the lowest eigenvalues of $\mathbf{K}_{t}$ at the corresponding points. The lowest eigenvalue of $\mathbf{K}_{t}$ is again computed at the immediate point, and equation (4.4) is repeated until a convergency satisfied.

\subsection{Automatic cutting and automatic increment technique}

The convergency problem is often arisen in computing the equilibrium path of arch structures. As mentioned above, $\Delta L$ in equation $(4,2)$ is a scalar initially chosen by the 
analysist. There is no rule for choosing this value, and a too large $\Delta L$ leads to failure of convergency. To overcome this problem, a technique similar to that used in [17] is adopted, in which a maximum number of iterations denoted nIM, is specified as an input data ( $\mathrm{nIM}=10$ for the present work). If convergency is not satisfied after nIM iterations, the iterative procedure is terminated, and the arc length is automatically cut by haft, so that the procedure is restated with a new length

$$
\Delta L_{n}=0.5 \Delta L
$$

Naturally, the convergency is more difficult for an increment where a high nonlinearity is present, and a shorter length is needed in this circumstance. To optimize the computation precess, a suitable length required for next increment is needed to compute. This purpose can be achieved by introducing the automatic increment technique adopted from [11], in which the length for next increment is computed by

$$
\Delta L_{n}=\Delta L \sqrt{\frac{\mathrm{nID}}{\mathrm{nIT}}}
$$

where $n I D$ is the desired number of iterations ( $\mathrm{nID}=5$ for the present work), and $n I T$ is the number of itcrations used in the present increment. As seen from (4.6), if $\mathrm{nIT}>\mathrm{nID}$, that means the nonlinearity is high for the present increment, and the next length is reduced by a factor of $\sqrt{\mathrm{nID} / \mathrm{nIT}}$.

Table 1. Elastic buckling load of clamped arch in Fig. 5 under concentrated load

\begin{tabular}{lcc}
\hline & B2ES element & B2ET element \\
\hline Present work & 0.8136 & 0.9142 \\
Belytschko and Glaum [13] & 0.82 & not available \\
Mirmiran et al. [19] & 0.806 & not available \\
\hline
\end{tabular}

\section{NUMERICAL INVESTIGATION AND DISCUSSION}

A computer code written in Matlab [18] has been developed based on the beam elements and discussed algorithm employed in this Section to analyze the instability phenomenon of some arches. For the iterative procedure, the Euclidian norm of the residual force vector is employed in the convergency criterion. A tolerance of $10^{-4}$ is employed in all the analyses.

\subsection{Accuracy verification}

This subsection aims to verify the accuracy of the elements and the numerical algorithm discussed above in the instability analysis of arch structures. To this end, we adopted herewith the clamped circular arch depicted in Fig. 5, previously investigated by Belytschko and Glaum [13], Mirmiran and co-workers [19]. For the sake of comparison, the data are kept in the US customary units as original, but their corresponding SI units are also given in the brackets as follows:

$$
\begin{aligned}
& R=133 \mathrm{in.}(3378.2 \mathrm{~mm}) ; \quad t=0.6 \mathrm{in.}(15.24 \mathrm{~mm}) ; \quad b=1.0 \mathrm{in} .(25.4 \mathrm{~mm}) ; \\
& L=34 \mathrm{in.}(863.3 \mathrm{~mm}) ; \quad h=1.1 \mathrm{in.}(27.94 \mathrm{~mm}) ; \quad E=10000 \mathrm{ksi}(68950 \mathrm{MPa}) .
\end{aligned}
$$

The arch has been discretized by 8 -equal elements, that is a total of 21 active degrees of freedom are employed. 
Table 1 lists the critical load of the clamped arch in Fig. 5 computed by the B2ES and B2ET elements. The load obtained by Belytschko and Glaum, Mirmiran et al. are also given in the table. It is noted that the critical load computed by the B2ES element in the present work is very much in agreement with that reported in $[13,19]$, where a mesh of 20 elements have been used. The higher value of the critical load obtained by the B2ET element might be caused by the lower order of the interpolation functions employed in developing the B2ET element as previous noted by the author in computing the buckling load of the beams [9].

The load-displacement curves of the clamped arch computed by different element types and a mesh of 8-equal elements are given Fig. 6 . In order to compare easier, and since the results in $[13,19]$ are described in the US units, the figure is illustrated in the same units. For the elasto-plastic analysis the yield stress and tangent modulus have been chosen as $\sigma_{0}=20 \mathrm{ksi}(137.9 \mathrm{MPa}), E_{t}=0$, as adopted by Mirmiran et al. The curves obtained by both the elastic and elasto-plastic analyses in the present work are very much in agreement with those reported in [19] using 20 curved beam elements.

'To demonstrate the convergency of the employed elements in mesh refinement, the computation has been performed with different meshes of 4, 8, 16 and 32 elements. Table 2 lists the critical loads of the elastic arch obtained by the B2ES and B2ET elements computed by various element numbers. The number of iterations used in the bracketing procedure described in Subsection 4.2 for obtaining the critical load is also listed in the table. As seen from the table, in the mesh refinement both the elements give the value of critical load which more comparative to that reported in the previous work $[13,19]$.

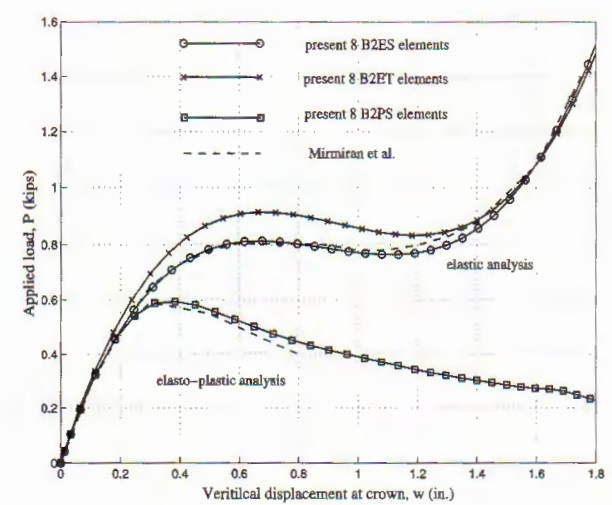

Fig. 6. Load-deflection curves for clamped arch computed by different element types

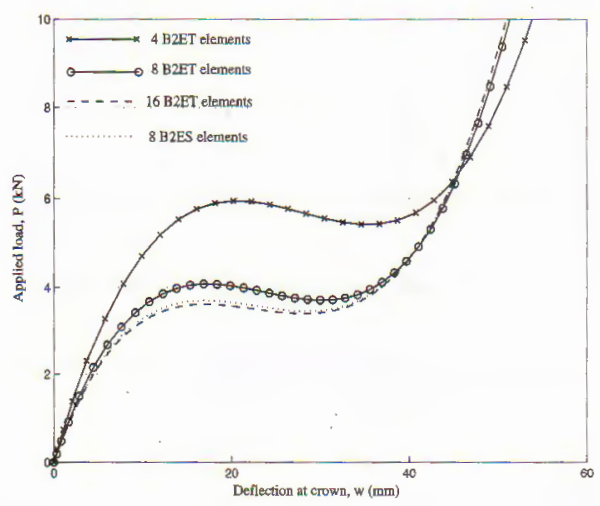

Fig. 7. Load-deflection curves for clamped arch computed by different element numbers

The load-deflection curves of the clamped arch in Fig. 5 computed by different element numbers are shown in Fig. 7. It is hardly seen the difference between the curves obtained by 4, 8, 16 and 32 B2ES elements, and only the curve computed by 8 B2ES elements is shown in the figure. There is no difference between the curves computed by 16 and $32 \mathrm{~B} 2 \mathrm{ET}$ element either. The load-deflection curve of the arch computed by the B2ET element converges to that obtained by the B2ES in the mesh refinement. It can be concluded from this Subsection that the co-rotational beam elements and the developed numerical algorithms are accurate in analyzing the instability phenomena of the arch structures. 
Table 2. Elastic buckling load of clamped arch in Fig. 5 computed by different element numbers

\begin{tabular}{ccccc}
\hline & B2ES element & \multicolumn{2}{c}{ B2ET element } \\
& $P_{c r}$ & $N_{i t}^{*}$ & $P_{c r}$ & $N_{i t}$ \\
\hline 4 elements & 0.8276 & 14 & 1.3327 & 18 \\
8 elements & 0.8136 & 16 & 0.9142 & 17 \\
16 elements & 0.8101 & 17 & 0.8282 & 17 \\
32 elements & 0.8056 & 15 & 0.8177 & 15 \\
\hline
\end{tabular}

* number of iterations

\subsection{Effect of rise to span ratio}

Fig. 8 shows the load-deflection curves for the clamped elastic arch having rise to span ratios $h / L$ in a range of $0.03-0.20$. All the curves in the figure are computed by 8-equal B2ES elements and shown in the dimensionless load-displacement space $\left(\frac{P L^{2}}{E I}, \frac{w}{L}\right)$. As shown in the figure, the more steeper arch the higher limit load is. The 'snap-through' phenomena tends to cease for more shallow arch and it almost disappears for the arch having the $h / L$ ratio of 0.03 .

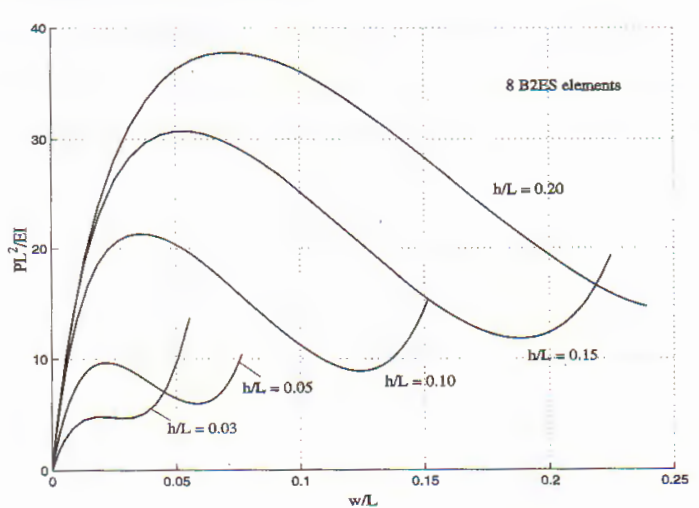

Fig. 8. Load-deflection curves for clamped elastic arch having different rise to span ratios

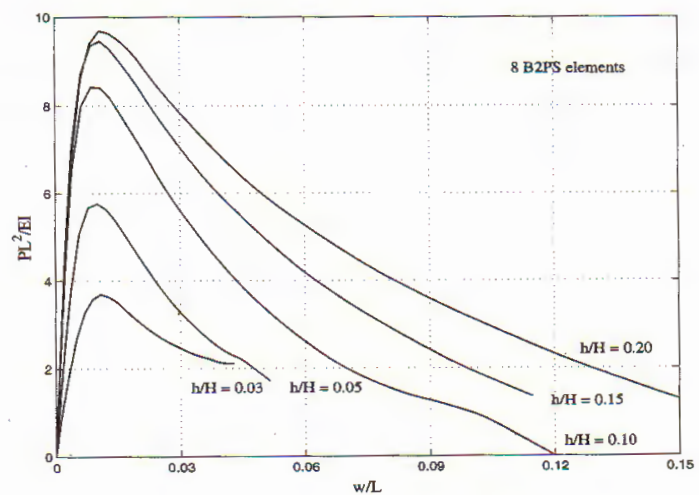

Fig. 9. Load-deflection curves for clamped elasto-plastic arch having different rise to span ratios

The load-deflection curves for the clamped arch having different rise to span ratios obtained by 8-equal B2PS elements are depicted in Fig. 9. The perfectly plastic material with yield stress the same as those in Subsection 5.1 is again adopted herewith. Besides the lower critical load, the behavior of the clamped elasto-plastic arch is similar to that of the elastic load, and this is very much in agreement with that reported by Mirmiran et al. in [19].

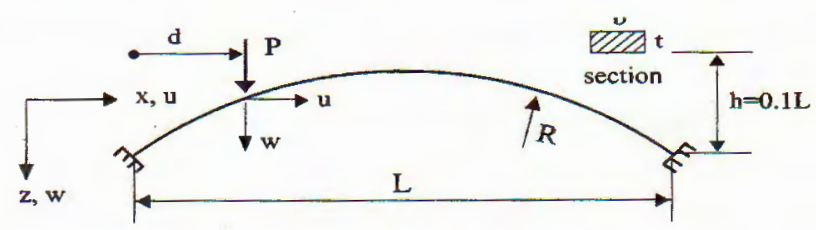

Fig. 10. Clamped arch under concentrated load at a point distanced $d$ from left support 


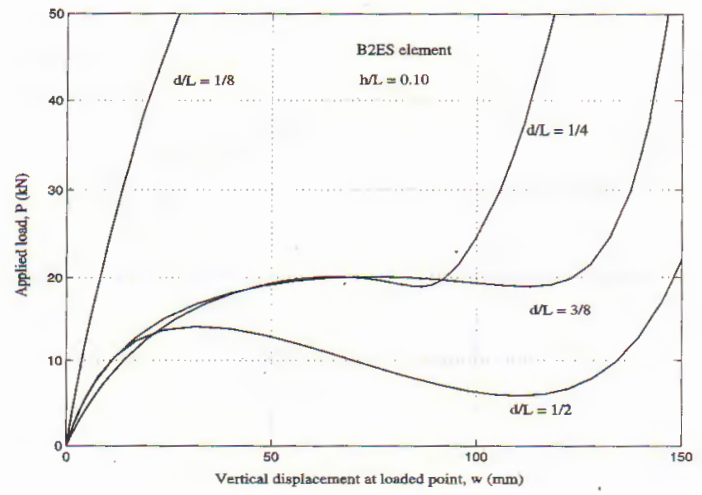

Fig. 11. Effect of loading pasition on vertical displacement at loaded point of elastic clamped arch

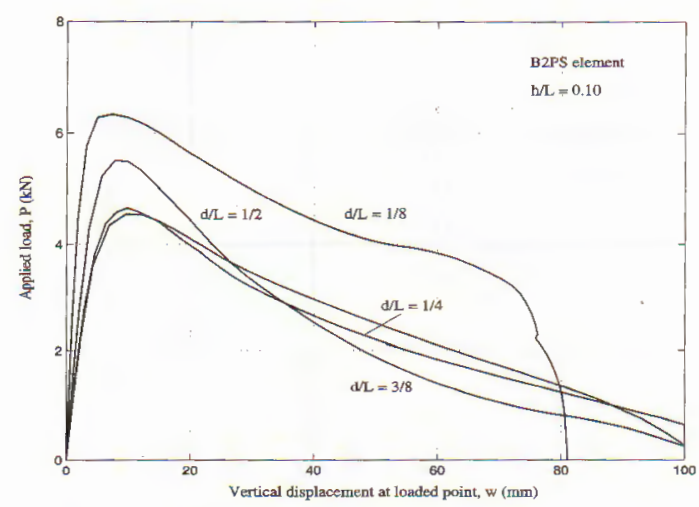

Fig. 12. Effect of loading position on vertical displacement at loaded point of elasto-plastic arch

\subsection{Effect of loading position}

To study the effect of loading position, we adopted herewith the arch in Subsection 5.1 , but with a rise to span ration $h / L=0.10$. Load $P$ is supposed acting at the point with a distance $d$ measured from the left support as shown in Fig. 10. The computation is performed for $d=1 / 8,1 / 4,3 / 8$ and $1 / 2$ using the B2ES and B2PS elements.

Fig. 11 shows the effect of loading position on the vertical displacement at loaded point of the elastic clamped arch, respectively. The corresponding figure obtained by elasto-plastic analysis is given in Fig. 12. All the analysis have been performed with a mesh of 8-equal elements. As seen in the figures, the post-buckling behavior of the arch when $d / L=1 / 2$ is very similar to that when $d / L=3 / 8$ for both cases of the elastic and elasto-plastic arch. When load $P$ is very near the fixed support, namely $d / L=1 / 8$, the arch is much more stable, and the critical load is much higher, irrespective of the analysis types.

\subsection{Effect of shear deformation}

Shear deformation effect can be investigated by introducing the slenderness parameter, defined as $[16,20]$.

$$
s=\frac{L_{o}}{\sqrt{I / A}},
$$

where $L_{0}$ is the length of the arch center line. The shear deformation becomes more important for the arch having lower value of slenderness parameter. For $s=100,200,300$ and 400 , keeping all the data of the clamped arch in Subsection 5.1, the computation is performed in this Subsection with $A=99.9086,399.6342,899.1770$ and 1598.5368 $\mathrm{mm}^{2}$ by using the B2ET element. The paths of elastic clamped arch at various values of slenderness parameter $s$ are depicted in Fig. 13. As clearly seen from the figure, a reduction in the critical load of the arch when lowering the slenderness parameter. The snap-through phenomenon gradually reduces when reducing the parameter $s$, and it almost disappears for $s=100$. In other words, the shear deformation plays an important role on the post-critical behavior of the arch. 


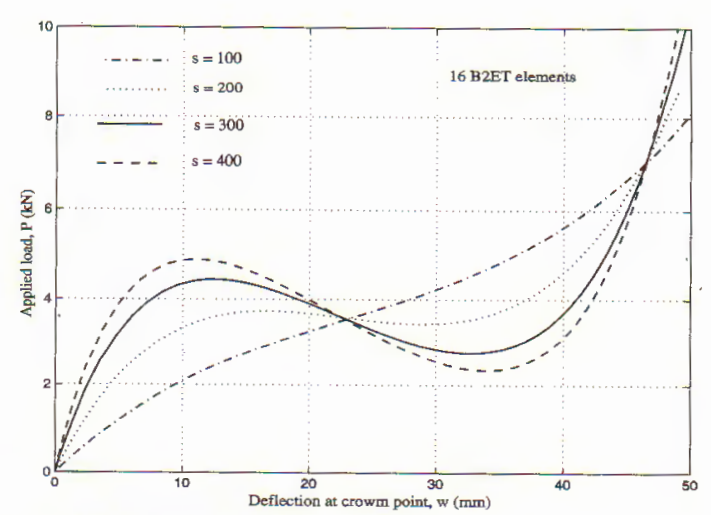

Fig. 13. Equilibrium paths for elastic clamped arch at various values of slenderness parameter

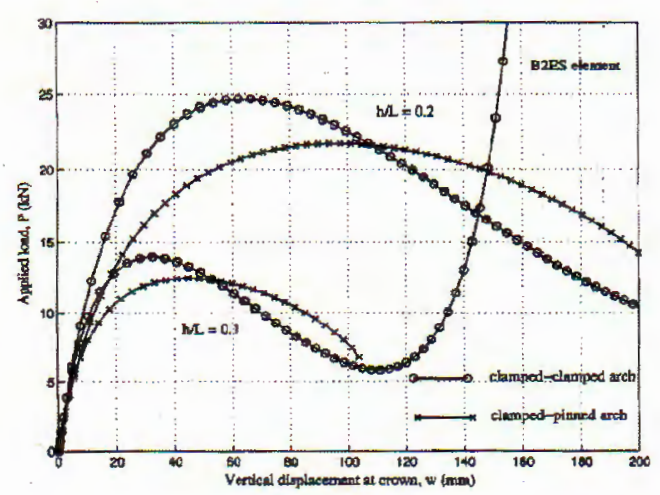

Fig. 14. Equilibrium paths for elastic arch with different boundary conditions

\subsection{Effect of boundary condition}

Two cases of boundary conditions are investigated: clamped at both ends, and clamped at one end and pinned at the other. The computation is conducted for the arch with a rise to span ratio of 0.10 and 0.20 using both the elastic (B2ES) and elasto-plastic (B2PS) elements. The elasto-plastic material is the same as that of Subsection 5.1. The computational results using the B2ES and B2PS elements are displayed in Fig. 14 and Fig. 15, respectively.

\subsection{Effect of hardening parameter}

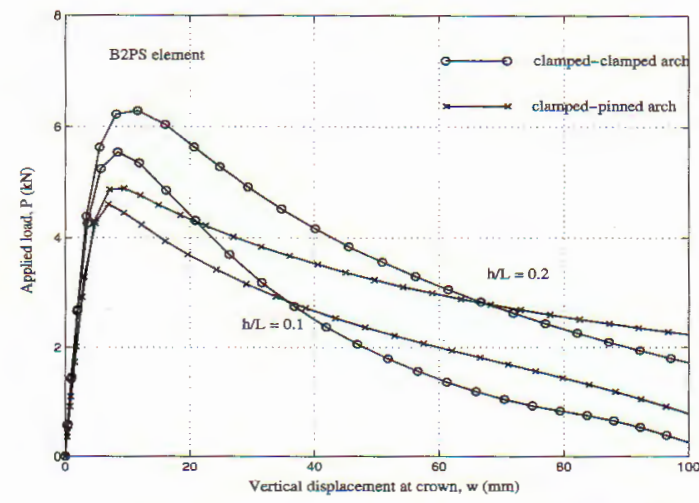

Fig. 15. Equilibrium paths for elasto-plastic arch with different boundary conditions

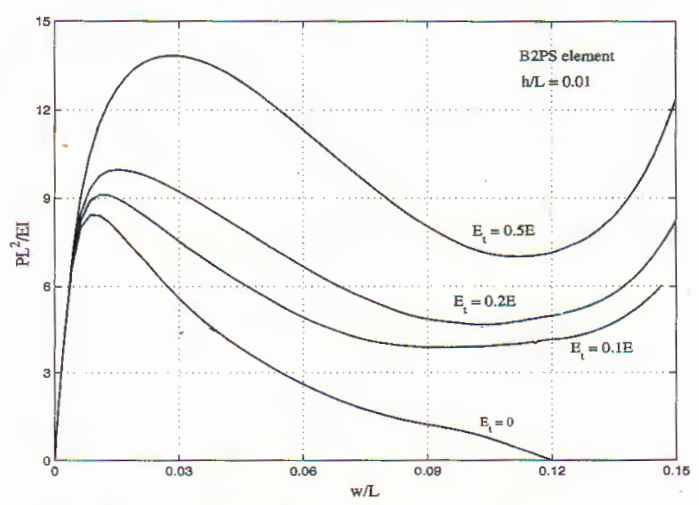

Fig. 16. Equilibrium paths for clamped elasto-plastic arch at various values of $E_{t}$

To investigate this effect, an arch with elasto-plastic geometry and material data as in Subsection 5.1, but with a rise to span ratio of 0.10 is adopted. The computation is performed with various values of tangent modulus of $E_{t}=0,0.1 E, 0.2 E$ and $0.5 E$, that is with hardening parameter $H=0,1111,2500$ and 10000 psi $(0,7661,17238$ and $68950 \mathrm{MPa}$ ), where $H$ is related to $E$ and $E_{t}$ by

$$
H=\frac{E_{t}}{1-\left(E_{t} / E\right)}
$$


Fig. 16 shows the equilibrium paths for the arch having various values of the tangent modulus $E_{t}$. As seen from the figure, not only the post-critical behavior of the arch but its critical load are strongly affected by the tangent modulus $E_{t}$, that is by the hardening parameter $H$ also. For the present arch, the yielding occurs before the limit point, and the hardening parameter affects the critical load: the higher hardening parameter the more critical load is. This result is different from the Euler beam previously investigated by the author in which the yielding occurs after the limit point and the hardening affects the post-critical behavior of the beam only.

\section{CONCLUDING REMARKS}

The paper has discussed how the instability problems of arches under quasi-static concentrated load can be analyzed by the finite element method. It has demonstrated that the arches can be accurately analyzed as equivalent framework using the co-rotational beam elements and the developed numerical procedure. The main conclusions obtained from the numerical investigation can be summarized as follows:

- Both the B2ES and B2ET beam elements can accurately access the critical load and model the post-critical behavior of arches, but the B2ET element requires finer mesh.

- The B2ET element is good in modelling the effect of shear deformation on the postcritical behavior of arch structures. The snap-through phenomenon tends to be disappeared for the arch having low slenderness parameter $s$. In other words, the shear deformation reduces the snap-through phenomenon in the large deformation behavior of arches.

- Yielding remarkably reduces the critical load and affects the post-critical behavior of arch structures. Hardening may increase the limit load and affect the post-critical behaviors of arches.

Acknowledgement. The present work has been partly financed by the National Program on Fundamental Research.

\section{REFERENCES}

1. A. Ghali and A. M. Neville, Structural Analysis, A Unified Classical and Matrix Approach, Third edition, E \& FN Spon, London, 1995.

2. R. G. Brockenbrough and F. S. Merritt (Editors), Structural Steel Sesigner's Handbook, McGraw-Hill, Inc., Second edition, New York, 1994.

3. R. D. Wood and O. C. Zienkiewicz, Geometrically nonlinear finite element analysis of beams, frames, arches and axisymmetric shells, Computers 83 Structures 7 (1977) 725-735.

4. Z. Xu and A. Mirmiran, Looping behavior of arches using corotational finite element, Computers \& Structures 62 (1997) 1059-1071.

5. M. A. Crisfield, Finite Elements and Solution Procedures for Structural Analysis. Volume 1: Linear Analysis, Pineridge Press, Swansea, 1986.

6. K. M. Hsiao and F. Y. Huo, Nonlinear finite element analysis of elastic frames, Computers 6 Structures 26 (1987) 693-701.

7. D. K. Nguyen and Q. Q. Do, Large deflections analysis of frames by elements contaning higer-order terms, Vietnam Journal of Mechanics 25 (2003) 243-254.

8. D. K. Nguyen and Q. Q. Do, Beam element for large displacement analysis of elasto-plastic frames, Vietnam Journal of Mechanics 26 (2004) 39-54. 
9. D. K. Nguyen, Effects of shear deformation on large deflection behavior of elastic frames, Vietnam Journal of Mechanics 26 (2004) 167-181.

10. Spring-Verlag, New York, Maple V, Language Reference Manual, 1991.

11. M. A. Crisfield, Non-Linear Finite Element Analysis of Solids and Structures, Volume 1: Essentials, John Wiley \& Sons, Chichester, 1991.

12. T. Belytschko and W. K. Liu and B. Moran, Nonlinear Finite Elements for Continua and Structures, John Wiley \& Sons, Chichester, 2000.

13. T. Belytschko and L. W. Glaum, Application of higher order co-rotational stretch theories to nonlinear finite element analysis, Computers 8 Structures 10 (1979) 175-182.

14. M. A. Crisfield, Non-Linear Finite Element Analysis of Solids and Structures, Volume 2: Advanced Topics, John Wiley \& Sons, Chichester, 1997.

15. M. A. Crisfield, A fast incremental/iterative solution procedure that handles 'snap-through', Computers \&5 Structures 13 (1981) 55-62.

16. D. K. Nguyen, Post-buckling behavior of beam on two-parameter elastic foundation, International Journal of Structural Stability and Dynamics 4 (2004) 21-43.

17. SAP2000, Analysis Reference, Version 7.0, Computers \& Structures Inc., California, U.A.S., 1998.

18. The Math Works Inc., Matlab, High-performance Numeric Computation and Visualization Software, Reference guide, 1992

19. A. Mirmiran, A. M Amde and Z. Xu, Elasto-plastic buckling of prestressed arches, International Journal of Structural Stability and Dynamics 2 (2002) 259-313.

20. S. P. Timoshenko and J. M. Gere, Theory of Elastic Stability, Second edition, McGraw-Hill, New York, 1961.

Received June 14, 2005

Revised September 14, 2005

\section{ĐốI XƯ MẤT ỔN ĐỊNH CƯA VÒM DƯớI TÁC ĐộNG CƯA LỰC TẬP TRUNG TỰA TĨNH}

Bài báo nghiên cứu các đặc tính mất ổn định của kết cấu vòm dưới tác động của lực tập trung tựa tĩnh. Vòm được rời rạc hóa như khung tương đương và được phân tích bằng phương pháp phần tử hữu hạn nhờ các phần từ dầm đồng hành phi tuyến. Thuật toán số, đặc biệt kỹ thuật tự động ngắt quá trình lặp và tính độ dài cung trong phương pháp lặp tăng dần đê tính lực tới hạn và đường cân bằng được trình bày chi tiết. Ảnh hường cùa hình học, điều kiện biên, biến dạng trượt và các tính chất vật liệu tới tới đối xử mất ổn định của vòm đàn hồi và đàn dèo được thảo luận kỹ lưỡng. 